\title{
AN OVERVIEW OF THE CONCEPT OF OPERATIONS FOR ASSEMBLY, INTEGRATION, TESTING AND GROUND SERVICING DEVELOPED FOR THE MPCV-ESM PROPULSION SYSTEM
}

\author{
M. Bielozer ${ }^{1}$ and B. Van Lear $^{2}$ \\ NASA Glenn Research Center, Cleveland, Ohio, 44138 \\ N. Kindred ${ }^{3}$ \\ Avatar Technologies, Kennedy Space Center, Florida, 32899 \\ And \\ G. Monien ${ }^{4}$ and U. Schulte ${ }^{5}$ \\ Airbus Space and Defense, Bremen, Germany
}

\begin{abstract}
A concept of operations for the Assembly, Integration and Testing (AIT) and the Ground Systems Development Operations (GSDO) of the European Service Module (ESM) propulsion system has been developed. The AIT concept of operations covers all fabrication, integration and testing activities in both Europe and in the United States. The GSDO Program develops the facilities, equipment, and procedures for the loading of hypergolic propellants, the filling of high-pressure gases, and contingency de-servicing operations for the ESM. NASA and ESA along with the Lockheed Martin and Airbus Space and Defense are currently working together for the EM-1 and EM-2 missions in which the ESM will be flown as part of the Orion Multi-Purpose Crew Vehicle (MPCV). The NASA/ESA SM propulsion team is collaborating with the AIT personnel from ESA/Airbus and NASA/Lockheed Martin to ensure successful integration of the European designed Service Module propulsion system, the Lockheed Martin designed Crew Module Adapter and the heritage Space Shuttle Orbital Maneuvering System Engines (OMS-E) being provided as Government Furnished Equipment (GFE). This paper will provide an overview of the current AIT and GSDO concept of operations for the ESM propulsion system.
\end{abstract}

\section{Introduction}

A FTER the cancellation of the Constellation Program in 2010, Congress continued to support the development of the Orion Multi-Purpose Crew Vehicle MPCV. The Space Launch System (SLS) is the heavy lift rocket which will send the MPCV to support long-duration deep space missions. The mission objectives which governed the architecture of the Orion MPCV include manned missions to an asteroid, the Moon, and Mars. As a backup capability, the MPCV is also planned to support cargo and crewed missions to the International Space Station (ISS).

The first flight, Exploration Mission (EM-1), for the Orion/SLS vehicle will be unmanned and is scheduled for the end of 2017. The main objective is to circle the moon and return safely to earth. The second flight of Orion, EM2, will be the first flight to carry astronauts. For Lunar missions, Orion launches on the SLS. The second stage, the

\footnotetext{
${ }^{1}$ SM Propulsion Engineer, In-Space Propulsion Systems Branch, M.S. 86-8, 21000 Brook Park Rd. Cleveland Ohio 44135

2 Orion Production Operations Engineer, Manufacturing Engineering Branch, 21000 Brook Park Rd. Cleveland, Ohio 44135.

${ }^{3}$ Spacecraft Integration Engineer, KLXSII, Kennedy Space Center, Florida 32899.

${ }^{4}$ AIT and Ground Operations Management, TSPOC4, P.O. Box 2861 56, 28631 Bremen, Germany.

${ }^{5}$ Operation Engineering Pneumatic \& Refueling Technologies, TEIB, P.O. Box 2861 56, 28631 Bremen, Germany.
} 1 American Institute of Aeronautics and Astronautics 
interim Cryogenic Propulsion Stage (iCPS), will insert Orion into a lunar trajectory. Orion will use its main engine to place itself into lunar orbit. Orion must maintain attitude control during lunar operations, perform the trans-Earth injection maneuver to return from the Moon, and perform entry, descent and landing.

The Orion MPCV consists of the Launch Abort System (LAS), the Crew Module (CM) and the Service Module (SM). In 2012 an Implementing Agreement with the European Space Agency (ESA), was signed which gave ESA the responsibility of designing and manufacturing the bulk of what makes up the $\mathrm{SM}$ and is referred to as the European Service Module (ESM). The SM also includes the Crew Module Adapter (CMA), the Spacecraft Adapter Jettisoned Fairings (SAJ), and the Spacecraft Adapter (SA) as shown in Figure $1^{1}$. The CM is

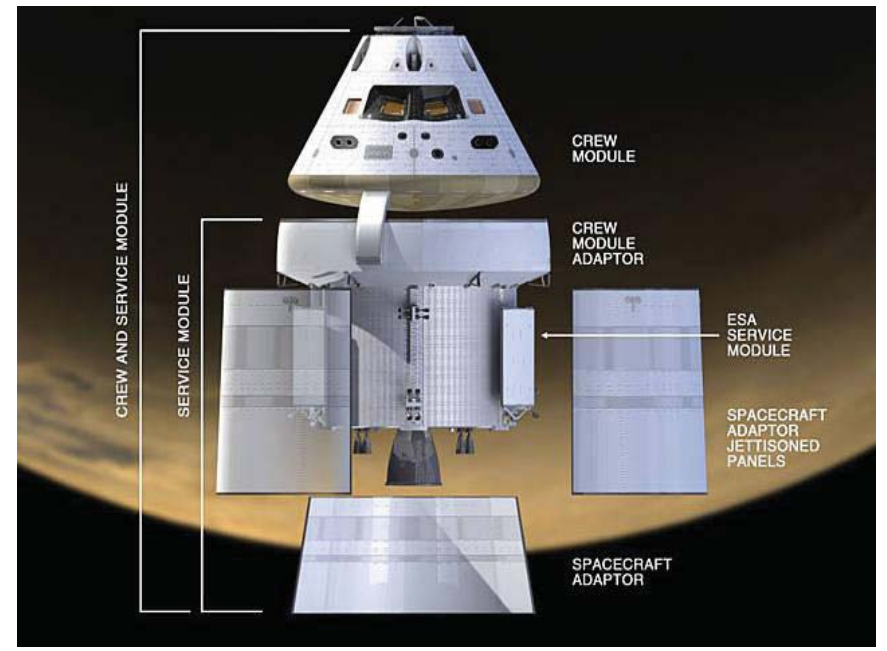

Figure 1. The Orion Multi-Purpose Crew Vehicle.

a capsule design, which consists of the primary structure for crew support, incorporates the bulk of the avionics systems, and provides the capability for atmospheric re-entry and a parachute landing. The LAS safely extracts the CM from the launch configuration in the event of an early launch abort. The SM performs in-space flight propulsion operations and power generation, and the SA is the structure that attaches Orion to SLS.

The concept of operations for Assembly, Integration, and Testing (AIT) and the ground servicing operations of the SM propulsion system, developed to a preliminary design review (PDR) level of maturity, will be described. A brief description of the basic manufacturing flow for the propulsion system is provided. An overview of the ESM propulsion system testing that will occur in both Europe and in the United States is outlined. Servicing operations for the propulsion system at the Kennedy Space Center (KSC) has also been defined. A general logistical mapping of the key ESM propulsion related test articles and the actual flight vehicle is summarized as well.

\section{Background}

Under the Constellation Program, Orion was to be designed and manufactured completely by Lockheed Martin. When the Implementing Agreement was signed, roles and responsibilities changed for the SM. ESA and their prime contractor Airbus Space \& Defense are responsible for designing, building, and qualifying the ESM for EM-1. As part of the development and qualification process, ESA will design and build a Structural Test Article (STA) which will be tested in Europe and at NASA's Plum Brook Station (PBS) in Sandusky, Ohio. They will conduct several propulsion development and qualification tests which will culminate in the Propulsion Qualification Model (PQM) testing held at the NASA White Sands Test Facility (WSTF). NASA and ESA will work together to ensure that the ESM design integrates with the other elements of the MPCV to deliver a qualified Flight Model (FM-1). Figure 2 is an illustration of the ESM test article and the flight article destinations prior to EM-1. ESA was also required, as part of the Implementing Agreement, to design the ESM so that it can be reproduced in the future. This means that the drawings and parts list will be provided to NASA. It has not yet been determined if ESA will produce the ESM for the EM-2 mission or the flights that follow ${ }^{2}$.

NASA's overarching responsibility is to develop the requirements for the overall MPCV vehicle to meet the mission objectives. As part of the Implementing Agreement, NASA agreed to provide several key elements to make the project successful. First, NASA is providing the SM main engine. The main engine is the heritage Space Shuttle Orbital Maneuvering System Engine (OMS-E) which will be repurposed for EM-1 and EM-2. These engines were removed and stored for future use after the Shuttles were retired. NASA will work with ESA to integrate the existing OMS-E into the ESM propulsion system. NASA is also providing the auxiliary engines for the ESM application. The engines will be procured from Aerojet Rocketdyne and delivered to ESA for integration into the vehicle. Another key part of the agreement was for NASA to provide and pay for the integrated hot-fire testing of the ESM propulsion system that will occur at WSTF. NASA will provide the facility, personnel, and the propellant. ESA will provide the test article. 


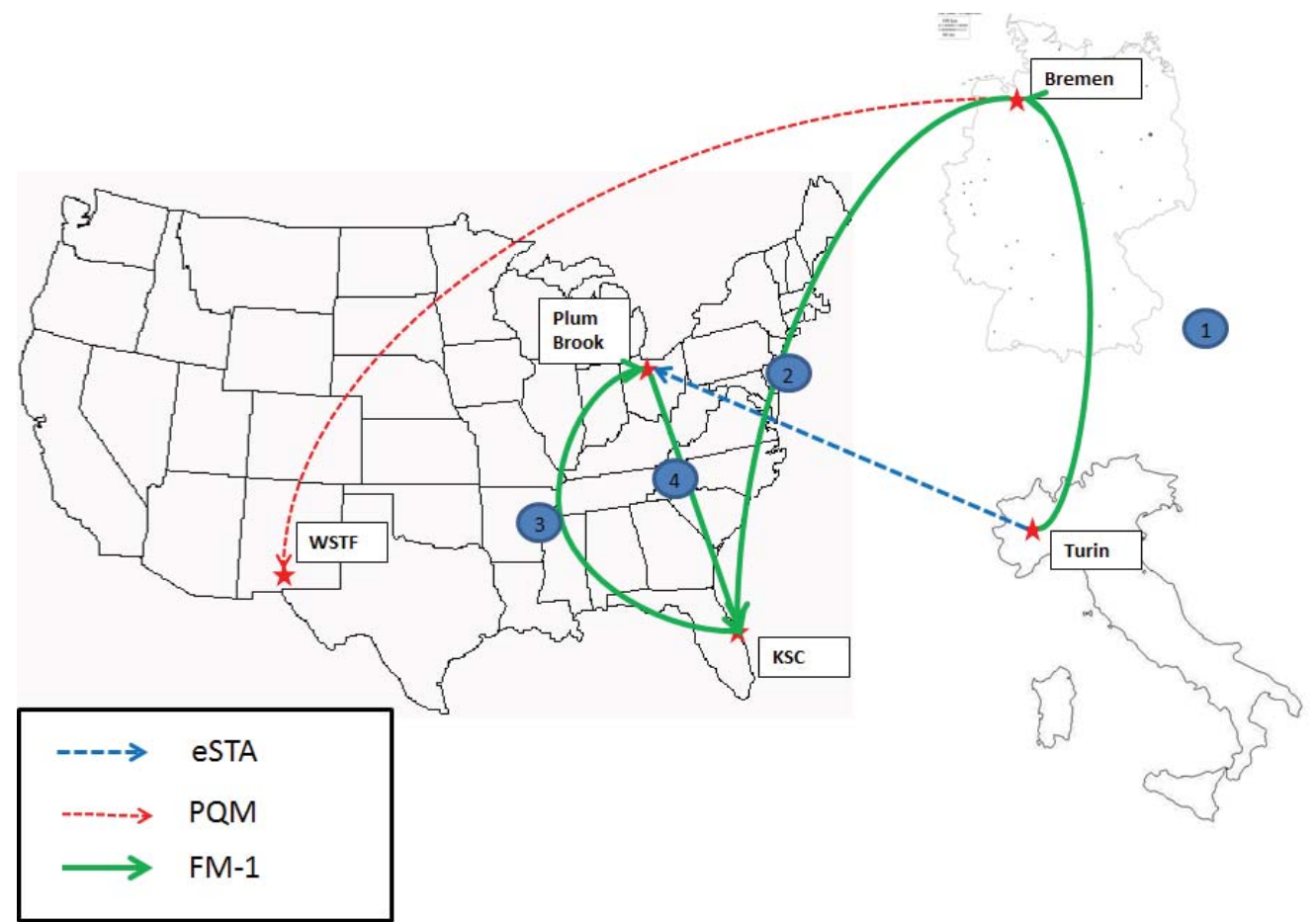

Figure 2. ESM test article and FM-1 destinations prior to flight.

Lockheed Martin is the overall integrator of the MPCV vehicle. They are responsible for ensuring the ESM interfaces properly with the CMA, SAJ, and the SA. They will receive the ESM from Airbus at the Operations and Checkout (O\&C) facility at KSC. After mating the ESM with the CMA, Lockheed will perform integrated functional tests. Lockheed will transport the CM and SM to and from PBS for environmental testing. They will then deliver the vehicle to NASA's Ground System Development Operations Program (GSDO). GSDO integrates the $\mathrm{CM} / \mathrm{SM}$ with the LAS, provides all ground servicing operations, and integrates Orion with the launch vehicle.

\section{Propulsion System Description}

The Orion SM propulsion system is a pressure fed, bipropellant system utilizing monomethylhydrazine (MMH) fuel and mixed oxides of nitrogen (MON-3) oxidizer. There are two oxidizer tanks, and two fuel tanks. Each pair of tanks is arranged in series. The fuel and oxidizer pressurization systems are kept fully separate to ensure there is no vapor migration between the two. Pressurization control is provided by a closed loop control system commonly referred to as a "bang-bang" control scheme. The propellant tanks feed three different engine types; one $6000 \mathrm{lbf}$ thrust gimbaled main engine with a thrust vector control system, eight $110 \mathrm{lbf}$ thrust auxiliary engines, and twentyfour $50 \mathrm{lbf}$ thrust Reaction Control System (RCS) engines. Isolation valves in the system allow different sets of engines to be isolated from the common feed system. Figure 3 is a simplified schematic of the SM propulsion system presented at PDR.

The SM propulsion system's fluid interfaces for ground servicing are located in two service panels on the CMA. The SM propulsion system fuel and oxidizer service panels are separated to comply with safety requirements. Each panel contains a fill/ drain valve for propellant, a vent/ullage valve, and a fill/drain valve for the high-pressure helium. The fill/drain lines for the propellants are routed from the CMA to a location near the bottom of the downstream propellant tanks. The vent/ullage lines connect directly into the top of the upstream propellant tanks. The fuel panel contains the fill/vent valve for the OMS-E pneumatic pack (PnP) high-pressure nitrogen gas which is used to fire the engine. In addition to these service valves, each panel contains four test ports which are used to fill/drain pad pressure helium gas in the various sections of the propulsion feed system. Each panel also contains a test port for the burst disk relief valve.

Airbus has included a number of test ports located in other locations on the ESM which can be used for functional testing of the SM propulsion system. These valves can only be accessed during the processing flow when 
the SAJ's, more commonly known as the fairings, are not installed. These test ports will be primarily used for functional leak checks, relief valve checkouts, and checkout testing of the OMS engine. Four valves located at the aft end of the SM are for contingency draining operations. These valves are not needed for servicing or testing, but are localized low points which will accelerate the propulsion system decontamination process. ${ }^{3}$

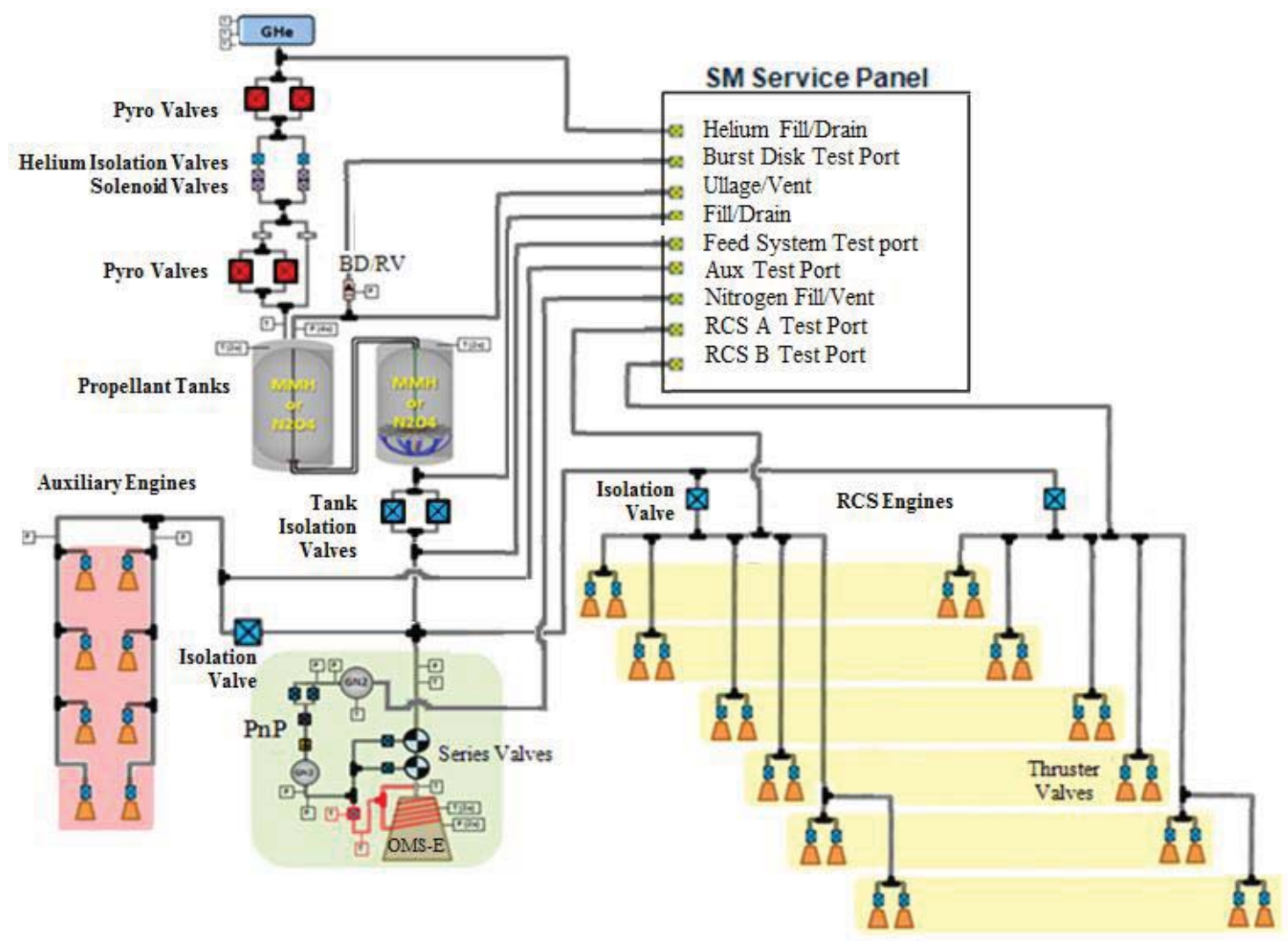

Figure 3. The ESM propulsion system.

\section{Assembly Integration and Test}

\section{A. ESM Structural Test Article}

The ESM Structural Test Article (eSTA) will be used to qualify the ESM structure design, and is the first major test article where propulsion system components are involved. The test article will consist of the flight like structural elements which make up the ESM, CMA, SAJ, and SA. The ESM structure will be built and assembled by a subcontractor of Airbus in Italy. The other elements will be provided by NASA/Lockheed Martin and manufactured in the US. There are two phases of testing planned for the eSTA. The first phase is a static test in Europe, and the second phase is a dynamic test in the US.

Before the ESM portion of the test article is sent to the US for testing, ESA will run a static test of the SM. Structural representatives will simulate the CMA and SA. A testing facility, located in Italy, will be used for this static test. The eSTA will not only have a flight like primary structure but it will also include critical mass simulators. The most important mass simulators on the eSTA will be the four flight-like propellant tanks. These tanks will be filled with a fluid simulant, in the US, for the dynamic testing phase. The main engine is also another mass simulator which will be included in the eSTA. NASA will loan a non-functioning OMS engine to Airbus. The engine was previously used for qualification purposes for the Shuttle Program. An additional test objective for the eSTA in Europe is to develop installation procedures for the OMS-E. The eSTA will include attachment points for the OMS-E, TVC, and mockup fluid connections. Along with the engine, NASA will loan OMS-E ground support equipment (GSE). NASA is providing ESA with the requirements and procedures that were used to install and checkout the OMS-E in the Shuttle OMS Pod. These procedures will help Airbus design their own procedures for installing the engine into the ESM. 
After the ESM structure has completed the static testing and has been outfitted with all the mass simulators, it will be shipped to the NASA's test facility at Plum Brook Station. There, the ESM will be assembled with the Lockheed Martin flight like representatives of the CMA, SA, and SAJ. Lockheed Martin will also provide a CM and LAS mass simulator which will be attached on top of the eSTA. The eSTA will be subjected to dynamic and pyro shock testing.

\section{B. Propulsion System Qualification Models and Test Activities}

In parallel to the eSTA activities, Airbus will develop and qualify the ESM propulsion system. At the time of the ESM PDR, three distinct test articles or qualification models will be used to qualify the propulsion system. The first two testing activities will be conducted in Europe at Airbus facilities. Hydraulic Model (HM) 1 will be used to conduct basic flow tests with a simulant fluid in order to understand pressure drops in the feed system, mixture ratios at engine flow control valves for optimum performance. The test will also validate flow parameters in Airbus's performance models. Transient behaviors like water hammer during system priming and when closing valves will also be analyzed. Transient pressure spikes as a result of interactions that may occur from opening and closing multiple valves in various frequencies will also be evaluated. The model will not include a pressurization control assembly (PCA) or propellant tanks. The facility will supply model with propellant simulants at pressure. Only the fuel side of the propellant feed system will be represented. The engines or thrusters will be represented by flow control valves, and the test article also includes the feed system isolation valves.

HM-2 will build off of the knowledge gained in HM-1. For HM-2, the PCA and propellant tanks for the fuel side will be added to the test article. Tests will be performed to demonstrate and evaluate the subsystem priming sequence. The PCA performance, latch valve stability, and time to achieve operational readiness will all be demonstrated. Pressurant and propellant flow stability for various mission phases as well as surge pressures during priming will be evaluated.

The last qualification model planned by Airbus is the Propulsion Qualification Model (PQM). This test will again complement the data gained in HM-1 and HM-2 and thus achieve a final verification of the propulsion subsystem requirements which require hot-fire testing. The Implementing Agreement allows Airbus to use NASA's White Sands Test Facility for the hot-fire test campaign.

The PQM test article will be built in Germany and then transported to the United States for testing. The PQM is a self-contained flight representative test article with on-board avionics, propellants, and pressurant gases. The configuration will include both the fuel and oxidizer fluidic networks and all feed system isolation valves. The propellant and pressurant tanks will be flight-like hydraulically, but will be more structurally robust to handle the stress of the testing campaign. Facility supplied helium gas for pressurizing the test article will also be available if testing requires it. A PCA for both the fuel and oxidizer will be present, but the level of redundancy has not yet been determined. NASA will provide a flight qualified OMS engine to Airbus for the PQM. The fluidic interfaces between the OMS-E and the PQM are intended to be as flight like as possible. The PQM will have a full complement of 8 auxiliary engines. Only one string (12 engines) of the RCS system is planned for the PQM, but future work may drive the test article to include additional engines. The Propulsion Drive Electronics (PDE) box is the avionics interface with the propulsion system. By using a flight like PDE and qualified software with the flight like valves, a near end-to-end qualification of the propulsion system is achieved.

The testing will occur in the 300 area of WSTF. The ambient test stand was used to test the Apollo crew and service module propulsion systems. The facility's most recent major testing campaign was for the Fleet Leader Test Program for the Space Shuttle. Modifications to the test stand in preparation for the hot-fire testing are currently underway. The facility engineers will design the test stand to fit the mechanical and electrical interfaces of the test article. The test stand has several levels and is equipped with both a removable enclosure and a flame bucket to route the exhaust gases. The helium and nitrogen pressurization supply system as well as the propellant storage and conditioning systems will be updated. The test stand will also undergo a rigorous structural assessment and modifications will be made to interface with the PQM structural attachment points. The electrical interfaces as well as the data collection system will be designed to meet Airbus requirements.

There are several major test objectives of the PQM test campaign. The PQM test is the only test entry during which integrated propulsion subsystem operation will be conducted with hypergolic propellants. The priming sequence, pyro activation and the resulting slam-start procedure will be demonstrated. System performance during simulated mission profiles will be monitored. Pressure drops across all feed line sections will be measured. The flow performance will be determined under hot-fire conditions which will lead to the finalization of the Airbus Mathematical Flow Model. Subsystem hydraulic shocks or water hammer effects will be characterized throughout the testing campaign. The hydraulic shocks caused by the various isolation and thruster valves operating together in mission sequences will be measured. The electrical equipment within the propulsion subsystem will also be verified 
during simulation runs with respect to the PCA performance and during degraded mission scenarios. Since the PQM testing is done at sea level, the thruster performance measurements are not a primary objective of the test series and no direct thrust measurement capability will be included in the test facility. However, propellant inlet pressure, temperature, and thruster chamber pressure will be measured and used to calculate the expected thruster performance. Another secondary test objective will be the verification of ground loading and deservicing operations. The PQM will include flight like servicing interfaces and will provide the program with the opportunity to demonstrate or potentially verify certain ground operations. After the testing campaign is concluded, the PQM will be decontaminated and stored at WSTF to enable future testing if required. ${ }^{4}$

\section{Flight Model 1 Assembly, Integration and Test Activities}

The ESM Flight Model 1 (FM-1) will be provided for integration, assembly and test into the Exploration Mission 1 (EM-1) article. The ESM structure will be manufactured, partially assembled and integrated in Italy. After shipping to Bremen, Germany the assembly and integration will be finalized as well as the functional tests of the ESM module.

Upon arrival in Bremen, the lower tank platform structure is separated from the upper module structure to facilitate parallel processing. Equipment installed on the lower tank platform includes the propulsion system auxiliary engines, their feed lines, and valves for both the auxiliary and RCS engines. The final element integrated into the lower tank platform structure is the OMS engine. All propulsion tubing is orbital arc welded and nondestructively inspected (NDI) using X-ray. Propulsion equipment installed in parallel on the upper module structure includes the helium tanks, PCA, and associated tubing.

After parallel upper and lower platform integration operations are complete, the two elements are re-assembled. The main propulsion tanks and $\mathrm{Z}$ and $\mathrm{Y}$ axis RCS pods are then installed, and the remaining propulsion system tubing is welded, inspected, and tested for leaks. The OMS nozzle will be installed, leak tested, and used during TVC testing to provide the proper inertial characteristics. The nozzle is then removed since the ESM shipping container cannot accommodate the ESM with the nozzle attached. After completion of the assembly and integration of the remaining ESM equipment, functional tests of the propulsion system will be performed. These tests include electrical tests of electronics, sensors, heaters, and valves; pneumatic tests will be conducted to leak check the system. Combined pneumatic and electrical tests will check the function of the valves under pressure. After completion of the propulsion and other subsystem testing, the ESM will be installed into its shipping container and transported to the US.

The ESM will arrive at the O\&C building at NASA KSC where it will be removed from its shipping container and installed on a transportation pallet. Airbus will perform the post-shipment health checks. Lockheed Martin will then perform the integration and test work in the US, except for equipment that interfaces only with the ESM that Airbus installs. The ESM will be placed onto an installation tool which is used to perform the structural interface mate between the ESM and the CMA. After the structural mate has been made the installation tool is removed and the assembled SM is moved to a clean room where tubing and electrical interfaces between the ESM and CMA are made. The tubing interfaces include fill and drain lines for propulsion system commodities as well as several test ports. Proof pressure and leak tests are then performed on the connected tubing, and functional checkout of the SM is performed. The OMS engine nozzle is then re-installed and leak tested by Airbus, and the SA cone is mated to the SM. This completes the SM standalone assembly, integration and test activities.

The CM is mated to the SM and the umbilical that transfers power, data, and commodities between the two modules is installed and tested. The first Orion system tests are then performed on the integrated CM, CMA, and ESM. The Short Stack (integrated CM and SM) is lifted onto a transportation fixture and rotated 90 degrees for horizontal transportation.

The Short Stack is air-transported from KSC to the NASA Plum Brook Station test facility for environmental testing. Upon arrival, the Short Stack is rotated back to the vertical orientation. The Solar Array Wings (SAW) are then installed and functional tested by Airbus. SM fairings, LAS simulator and closeouts are installed. The vehicle is moved to the acoustic test chamber where post-shipment functional and pre-environmental performance testing is performed. The vehicle is subjected to acoustic testing and post-test functional testing. The LAS, closeouts, fairings and ESM SAWs are then removed in preparation for the thermal vacuum test. The Short Stack is transported to the vacuum chamber where thermal balance, vacuum, and functional tests are performed first. Electromagnetic interference testing will be performed last. Finally, the vehicle is re-installed on its shipping fixture and rotated for horizontal transportation.

The Orion Short Stack is shipped back to the O\&C building at KSC. After the vehicle is re-oriented to the vertical position, the SAWs are re-installed by Airbus. The SM fairings are also re-integrated and final closeouts are 
installed. A final integrated system test is performed, and then the processing responsibility and ownership of the vehicle is transferred to the Ground System Development Operations Program (GSDO).

\section{Ground Operations}

When the flight vehicle leaves the O\&C Facility at KSC, GSDO assumes responsibility for the ground operations leading up to and including launch. GSDO is responsible for preparing the SLS launch vehicle, the second stage iCPS, the LAS, and the MPCV Short

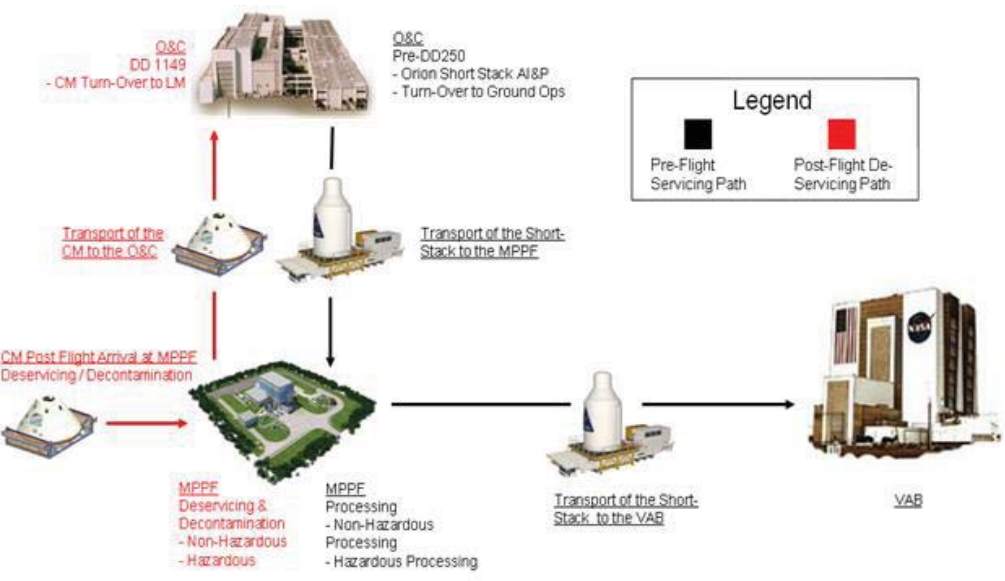

Figure 4. The GSDO processing flow for MPCV. Stack. The elements are assembled in the Vertical Assembly Building (VAB) and then transported to the launch pad. The processing of the MPCV Crew and Service Module (CSM) takes place in the Multi-Payload Processing Facility (MPPF). The generic GSDO processing flow for the CSM is shown in Figure 4.

The MPPF has been allocated to the MPCV Program with the potential for shared operations with commercial customers. The MPPF provides the capability to perform offline, integrated, spacecraft hazardous and nonhazardous processing in an environmentally controlled, Class 100,000 clean room ${ }^{5}$. Performing the CSM hazardous servicing offline will reduce risk to the integrated flow at the VAB and launch pad. This ensures greater launch availability. The offline processing facility will provide portable and permanent access stands, Command Control and Communication (CCC) equipment, and Ground Support Equipment (GSE). The facility utilizes a hypergolicvent and scrubber system, a hazardous gas detection system and a water deluge system for hazardous processing.

Non-hazardous commodity operations will be conducted first and will include filling gaseous nitrogen (GN2) and gaseous helium $(\mathrm{GHe})$ pressurants for the propulsion system. The MPPF also services the GN2 and gaseous oxygen (GO2) commodities for the ECLSS system. HFE 7200 servicing will also be conducted for the SM coolant system.

The hazardous propellant loading operations are conducted last. The CSM propellants (hydrazine, MMH, and MON-3) are loaded serially. Bulk propellant is transferred to the MPPF in Department of Transportation (DOT) Generic Propellant Transfer Units (GPTU) on a just-in-time basis.

The ground-to-vehicle interfaces will be mated and leak checked in the preparatory operations. Pressure across the vehicle fill/vent valves will be balanced using GHe. The SM fill/vent valves will then be opened. A blanket pad pressure is applied to the SM propulsion feed system. This pad pressure will prevent moisture intrusion into feed system during ground processing. With the propellant feed system pad pressure established, the SM propellant tank will be pressurized in preparation for liquid transfer.

With the propellant source on line, propellant samples will be taken from the panel sample ports on these GSE legs. These samples will be expedited for analysis of purity and particulate. Once the sample results are confirmed to meet requirements, loading operations can commence.

The GPTU's are pressurized to match pressure across the SM interface valves. Load cells measure the weight of each GPTU throughout the loading process. An initial tare weight measurement of the GPTU's is taken, and then the fill/drain valves are opened to bring the GSE fluid circuit on-line with the tanks. The liquid flow rate is restricted to for the first $1 \%$ of load process. This ensures that the liquid acquisition device and several inches of the screen will be submerged before the flow rate is increased. After $1 \%$ of the propellant is filled, the flow rate will be increased for the remainder of the load. Pressure in the tank during liquid transfer will be maintained by opening and closing the GSE vent valve. During liquid transfer, the load cell measurements of the GPTU's will be monitored until the target totalized delta weight is achieved (tolerance of $+/-0.2 \%$ ).

At this point, tank venting is terminated and the fluid fill/drain valve is closed. The propellant tanks are then pressurized to a flight pressure of 15 bar (218 psia) through the tank vent valve. When pressure is stable in the propellant tanks, the vent interface valve is closed. The interfaces are evacuated and pulse purged to safe the ground interfaces for disconnect. The GSE is disconnected from the SM servicing interfaces, and the flight caps are then installed in preparation for launch. The SM propellant system is now in configuration for flight and ready for 
integration with SLS. The spacecraft will return to the MPPF for a preflight contingency which requires deservicing of the CSM propellants. After successful MPPF servicing operations, the Short Stack is transported to the VAB where it is integrated with the LAS and SLS. After stacking operations are complete, MPCV and Orion travel out to the launch pad on top of the mobile launcher.

\section{Conclusion}

An AIT and ground operations concept of operations for the ESM propulsion system has been established for the preliminary design review. The propulsion system will be designed and qualified through an international partnership between the NASA and the European Space Agency. Work has already begun on the eSTA and the PQM. Both test articles are scheduled to arrive in the US in 2015 and 2016 respectively, and the flight model ESM for the EM-1 mission will arrive in 2016. The plans developed thus far will lay the foundation for the fabrication, qualification and final processing of America's next manned spacecraft.

\section{Reference}

${ }^{1}$ Angelillo, P., “MPCV-ESM Design and Development Plan,” MPCV-RIBRE-PL-005, March 2014.

${ }^{2}$ Witte, H., "MPCV ESM Assembly, Integration and Test Plan," MPCV-RIBRE-PL-0025, March 2014.

${ }^{3}$ Schulte, U., "MPCV-ESM Fluidic Ground Support Equipment Specification for PSS Acceptance Testing," MPCV-RIBRESPE-0050, March 2014.

${ }^{4}$ Jager, M., "MPCV-ESM Propulsion Subsystem PQM Test Plan,” MPCV-RIBRE-PL-0020, March 2014.

${ }^{5}$ Flemming, T. Facilities Handbook for Multi-Payload Processing Facility (MPPF), K-STSM-14.1.17- Basic-MPPF.May 1995. 